\title{
The effect of inbreeding on the redistribution of genetic variance of fecundity and viability in Tribolium castaneum
}

\author{
ALMUDENA FERNÁNDEZ, MIGUEL ANGEL TORO \& CARLOS LÓPEZ-FANJUL*† \\ CIT-INIA, Area de Mejora Genética Animal, Carretera de La Coruña, km. 7, 28040 Madrid, Spain and †Departamento \\ de Genética, Facultad de Ciencias Biológicas, Universidad Complutense, 28040 Madrid, Spain
}

Inbreeding was carried out to investigate the genetic properties of fecundity and viability (early and late) in a population of Tribolium castaneum. Heritability estimates in the base population were intermediate for fecundity and small and nonsignificant for viability. All traits showed strong inbreeding depression. For both viability traits, a significant increase of both within-line additive variance and between-line variance was observed with inbreeding. For fecundity, however, these changes were not detected. The results are compatible with the genetic variance of viability in the base population being generated by segregation of a number of deleterious recessive alleles at low frequencies. For fecundity, an additional class of loci with substantial additive effects must be postulated.

Keywords: artificial selection, fecundity, genetic variance, inbreeding, Tribolium castaneum, viability.

\section{Introduction}

The redistribution of the genetic variance of a neutral quantitative trait in a population subdivided into lines of equal effective size has been theoretically studied for different types of gene action. The cases of additive genes, recessives at low initial frequency and additive $\times$ additive epistasis have been considered by Wright (1951), Robertson (1952) and Goodnight (1988), respectively.

Summarizing, with additive action within and between loci, the between-line variance relative to the genetic variance in the base pópulation increases linearly with the inbreeding coefficient $\left(F_{t}\right)$ as $2 F_{t}$. The increase will be larger than that predicted by the additive model when part or all initial variation is caused by rare recessive alleles and/or by loci showing additive $\times$ additive epistasis. In parallel, the within-line additive genetic variance contributed by additive loci, also expressed as a proportion of the base population value, is expected to decrease with time as $\left(1-F_{t}\right)$. In the other cases, however, it can initially increase with inbreeding up to a maximum,

${ }^{*}$ Correspondence. and subsequently decrease to zero. Thus, genetic drift can convert nonadditive variance into additive variance which may contribute to the response to selection (Goodnight, 1987, 1988; Tachida \& Cockerham, 1989). This phenomenon is particularly relevant to the efficacy of the interdemic selection phase of Wright's shifting balance theory of evolution (Wright, 1978; Wade \& Goodnight, 1991) and to the response to artificial selection in structured populations (see López-Fanjul, 1989, for a review).

Experimental data supporting this conversion of nonadditive into additive variance are scarce (see Carson, 1990, for a review). In Musca domestica, Bryant et al. (1986) found an increase of the additive variance of several morphological traits after lines passed through a single-generation bottleneck. The statistical significance of this result has been questioned by Lynch (1988). In Drosophila melanogaster, López-Fanjul \& Villaverde (1989) and García et al. (1994) obtained a significant increase of both the additive variance and the heritability of egg-to-pupa viability after one generation of brother-sister mating.

The objective of the present experiment is to explore the situation for life-history traits in Tribolium castaneum, following an experimental design 
analogous to that used by López-Fanjul \& Villaverde (1989).

\section{Materials and methods}

The Consejo population was captured near Madrid and has been maintained in this laboratory since 1964 in ten bottles. A circular mating scheme was used to ensure a sufficiently large population (about 1000 potential parents per generation). All beetles in this experiment were kept in the same dark incubator at 70 per cent relative humidity and $33^{\circ} \mathrm{C}$. The culture medium consisted of 95 per cent whole wheat flour and 5 per cent dried powdered brewer's yeast by weight.

Three fitness component traits were considered: (1) 2-day egg-laying of mated females, individually scored from the 11th to the 13th day after adult emergence; (2) early viability, measured individually as the ratio between the number of eggs laid by a female during the period mentioned and the number of larvae present 2 days later; and (3) late viability, measured in the same way as for early viability but with respect to the number of larvae present 11 days later. Both viability traits were expressed in percentages.

Thirty-two lines, 16 inbred $\left(F_{0}=0.25\right)$ and 16 noninbred $\left(F_{0}=0\right)$ were established. For the three traits, the mean, between-line variance and withinline additive genetic variance in both types of lines were compared.

\section{Noninbred lines}

From the Consejo population, a sample of 160 males and 160 virgin females was collected, mated at random in pairs, and divided into 16 groups of equal size. From each group, both a selected and a control line were started, these treatments being assigned at random with the restriction of there being eight lines of each type. One generation of upward selection was carried out independently for each of the three traits considered. To do this, three sets of two male and two female offspring were obtained from each of the 10 original pairs in a group. In each set, 20 random matings were established and a different trait scored (generation 0). The five highest scoring pairs were selected as parents and each contributed four male and four female offspring. These 20 males and 20 females were mated at random and the resulting pairs were evaluated for the selected trait (generation 1). The control lines were maintained in the same way as the selected lines, but the three traits were evaluated on the same 20 matings per generation, and the five pairs of parents were randomly selected. Full-sib matings were always avoided in all lines and in all generations.

\section{Inbred lines}

Eight matings were randomly taken from the base population. From each mating, five offspring of each sex were taken and mated individually, each sib mating contributing 16 male and 16 female offspring. These 80 pairs were divided into four sets of 20 pairs each. From each set, a line selected for one of each of the three traits considered and a control line were started. From here, we followed the same procedure as in the noninbred lines.

In all, there were eight inbred and eight noninbred lines selected for each trait, along with their corresponding randomly selected control lines. All lines selected for the same trait (inbred or noninbred) and their companion control lines were maintained on a strictly contemporary schedule.

Divergent selection for fecundity or viability is expected to show negative asymmetry, as downward response will be mostly the result of an increase in the frequencies of all sorts of deleterious alleles present in the base population. Therefore, downward selection was not carried out in our experiment, as the response obtained would not be relevant to our purposes.

For each selected line and trait the following parameters were calculated: (1) selection differential $(S)$, as the difference between the mean of the selected parents and that of the population from which they were taken; $(2)$ response to selection $(R)$, as the difference between the means of the selected trait at generations 1 and 0 , expressed as deviation from that of the companion inbred control line or from the average of all noninbred control lines; (3) realized heritability $\left(h_{\mathrm{R}}^{2}\right)$, as the quotient between the response and the selection differential; (4) phenotypic variance $\left(V_{\mathrm{P} 0}\right)$ at generation 0 ; and $(5)$ additive genetic variance $\left(V_{\mathrm{A} 0}\right)$ at generation 0 , as the product of the realized heritability by the phenotypic variance. Standard errors of average phenotypic and additive variances over lines were estimated empirically. Those of the between-line component of variance were calculated following the procedure outlined by Lynch (1988). Only calculations on untransformed data are presented as the conclusions were not substantially modified after an arcsine transformation was performed.

Avoidance of full-sib mating (except in the formation of the inbred lines) ensured that the rate of 
inbreeding was negligible during the period considered (less than 0.01 per generation) both in the control and the selected lines. Thus, the inbreeding coefficient of selected parents and their offspring was approximately the same. This was deemed important, as viability can in principle be regarded as a property both of the mother and of the offspring. In Drosophila melanogaster, viability seems to be largely dependent on the maternal genotype (see Chapco, 1979, for a review), but this property has not been investigated in Tribolium. We have considered viability as a trait of the mother, which implies that we were carrying out individual selection. On the other hand, if viability had depended entirely on the larval genotype, family selection would have been practised and the realized heritability would have been the heritability of full-sib family means. This does not affect the significance of the difference between the realized heritabilities estimated in inbred and noninbred selected lines, as this significance has been tested empirically.

\section{Results}

Table 1 shows the effects of inbreeding on the mean, and on the within- and between-line components of the phenotypic variance for egg-laying and both viability traits in the control lines.

In each type of control line (inbred and noninbred), the difference between the means at genera- tions 0 and 1 was not significant for all traits. However, significant differences were found between the means of inbred and noninbred control lines $(P<0.05)$. The rate of inbreeding depression per 10 per cent increase in inbreeding coefficient was 2.6 eggs, 1.7 larvae and 4.2 larvae for fecundity, early viability, and late viability, respectively (about 4-5 per cent of the noninbred mean). On the average, both the within- and the between-line components of the phenotypic variance considerably increased with inbreeding for all traits. Increments in the within-line variance were significant in all cases; those corresponding to the between-line variance were only significant for late viability $(P<0.05)$.

Table 2 shows the average values of $S, R, V_{\mathrm{P}}, h_{\mathrm{R}}^{2}$ and $V_{\mathrm{A}}$ for each trait and type of line. For fecundity, all those values were significantly different from zero $(P<0.05)$ both in inbred and noninbred lines. However, in no case was a significant difference detected between these two sets of lines. On the other hand, the behaviour of both viability traits was very similar and strikingly different from that observed for fecundity. The average selection differential was higher in inbred than in noninbred lines, reflecting a parallel increase in the phenotypic variance. Selection resulted in a negative response on average in the noninbred lines (not significantly different from zero). However, the response to selection in the inbred lines was positive and significant for both traits. Consequently, the additive genetic variance of

Table 1 Mean, within-line $\left(V_{\mathrm{W}}\right)$ and between-line $\left(V_{\mathrm{B}}\right)$ components of the phenotypic variance for fecundity and viability of randomly selected control lines of Tribolium castaneum in two consecutive generations $(t)$

\begin{tabular}{llcccc}
\hline Trait & Type of line & $t$ & Mean & $V_{W}$ & $V_{B}$ \\
\hline Fecundity & Noninbred & 0 & $44.3 \pm 0.6$ & $46.1 \pm 5.5$ & $1.1 \pm 1.9$ \\
& & 1 & $43.1 \pm 0.5$ & $36.0 \pm 4.5$ & $3.6 \pm 3.0$ \\
& & Average & $43.7 \pm 0.4$ & $41.1 \pm 3.6$ & $2.4 \pm 1.8$ \\
& Inbred & 0 & $37.1 \pm 0.7$ & $72.9 \pm 8.8$ & $3.4 \pm 3.9$ \\
& & 1 & $37.3 \pm 0.6$ & $55.6 \pm 6.7$ & $6.1 \pm 4.9$ \\
Early viability & Noninbred & Average & $37.2 \pm 0.5$ & $64.3 \pm 5.5$ & $4.8 \pm 3.2$ \\
& & 0 & $41.4 \pm 0.7$ & $78.5 \pm 9.4$ & $3.1 \pm 3.9$ \\
& & 1 & $41.7 \pm 0.8$ & $86.7 \pm 10.8$ & $4.5 \pm 5.0$ \\
& Inbred & Average & $41.6 \pm 0.5$ & $82.6 \pm 7.2$ & $3.8 \pm 3.2$ \\
& & 0 & $36.6 \pm 1.0$ & $149.1 \pm 17.9$ & $13.3 \pm 11.4$ \\
& & 1 & $38.0 \pm 0.8$ & $88.8 \pm 10.7$ & $16.6 \pm 11.4$ \\
Late viability & Average & $37.3 \pm 0.7$ & $119.0 \pm 10.4$ & $15.0 \pm 8.1$ \\
& \multirow{2}{*}{ Noninbred } & 0 & $80.9 \pm 1.1$ & $191.5 \pm 23.1$ & $7.7 \pm 6.7$ \\
& & 1 & $77.3 \pm 1.5$ & $308.9 \pm 38.5$ & $3.5 \pm 11.3$ \\
& Inbred & Average & $79.1 \pm 0.9$ & $250.2 \pm 22.4$ & $5.6 \pm 7.6$ \\
& & 0 & $70.1 \pm 1.6$ & $350.4 \pm 42.0$ & $44.2 \pm 33.8$ \\
& & 1 & $67.1 \pm 1.5$ & $294.9 \pm 35.4$ & $43.7 \pm 31.9$ \\
& & Average & $68.6 \pm 1.1$ & $322.7 \pm 27.5$ & $44.0 \pm 23.3$ \\
\hline
\end{tabular}


Table 2 Average selection differential applied $(S)$, response $(R)$, phenotypic $\left(V_{\mathrm{P} 0}\right)$ and additive $\left(V_{\mathrm{A} 0}\right)$ variances of the base population of Tribolium castaneum, and realized heritability $\left(h_{\mathrm{R}}^{2}\right)$ of the noninbred $\left(F_{0}=0\right)$ and inbred $\left(F_{0}=1 / 4\right)$ selected lines

Trait

\begin{tabular}{|c|c|c|c|c|c|c|}
\hline & \multicolumn{2}{|c|}{ Fecundity } & \multicolumn{2}{|c|}{ Early viability } & \multicolumn{2}{|c|}{ Late viability } \\
\hline & $F_{0}=0$ & $F_{0}=1 / 4$ & $F_{0}=0$ & $F_{0}=1 / 4$ & $F_{0}=0$ & $F_{0}=1 / 4$ \\
\hline$S$ & $8.0 \pm 0.9^{*}$ & $8.6 \pm 0.5^{*}$ & $9.9 \pm 1.0^{*}$ & $11.6 \pm 1.4^{*}$ & $11.6 \pm 1.1^{*}$ & $19.4 \pm 2.4^{*} \dagger$ \\
\hline$R$ & $4.7 \pm 0.6^{*}$ & $3.7 \pm 2.2 *$ & $-2.1 \pm 1.1$ & $4.7 \pm 1.7^{*}+$ & $-1.4 \pm 1.8$ & $7.3 \pm 2.4^{*} \dagger$ \\
\hline$V_{\mathrm{P} 0}$ & $40.2 \pm 9.6^{*}$ & $46.8 \pm 10.1^{*}$ & $98.9 \pm 22.5^{*}$ & $114.1 \pm 27.3^{*}$ & $158.8 \pm 24.9^{*}$ & $283.3 \pm 36.9^{*} \dagger$ \\
\hline$V_{\mathrm{A} 0}$ & $20.8 \pm 2.4^{*}$ & $18.8 \pm 8.4^{*}$ & $2.9 \pm 2.3$ & $42.9 \pm 12.5^{*} \dagger$ & $13.0 \pm 7.6$ & $107.7 \pm 31.7^{*} \dagger$ \\
\hline$h_{\mathrm{R}}^{2}$ & $0.50 \pm 0.20 *$ & $0.40 \pm 0.10^{*}$ & $0.03 \pm 0.02$ & $0.40 \pm 0.10^{*} \dagger$ & $0.08 \pm 0.05$ & $0.40 \pm 0.10^{*} \dagger$ \\
\hline
\end{tabular}

*Significantly different from zero $(P<0.05, t$-test $)$.

$\dagger$ Significantly different from estimate at $F_{0}=0(P<0.05, t$-test $)$

Table 3 Observed $(O)$ values of the change of the within-line additive variance $\left(V_{\mathrm{AF}}\right)$ and the between-line variance $\left(V_{\mathrm{BF}}\right)$ with $F=1 / 4$ inbreeding, together with expectation $(E)$ for $n_{1}$ additive and $n_{2}$ equivalent recessive loci initially segregating at frequencies $p_{1}$ and $p_{2}$, respectively, generating the additive variance in the base population of Tribolium castaneum $(F=0)$ and the inbreeding depression observed

\begin{tabular}{|c|c|c|c|c|c|c|c|c|}
\hline & \multirow[b]{2}{*}{$n_{1}$} & \multirow[b]{2}{*}{$n_{2}$} & \multirow[b]{2}{*}{$p_{1}$} & \multirow[b]{2}{*}{$p_{2}$} & \multicolumn{2}{|c|}{$V_{\mathrm{AF}} / V_{\mathrm{A} 0}$} & \multicolumn{2}{|c|}{$\left(V_{\mathrm{BF}}-V_{\mathrm{B} 0}\right) / V_{\mathrm{A} 0}$} \\
\hline & & & & & $O$ & E & $O$ & $E$ \\
\hline \multirow[t]{2}{*}{ Fecundity } & 24 & 43 & 0.50 & 0.10 & 0.90 & 0.91 & 0.12 & 0.57 \\
\hline & 25 & 135 & 0.07 & 0.07 & 0.90 & 0.89 & 0.12 & 0.55 \\
\hline Early viability & - & 29 & - & 0.07 & 14.79 & 13.80 & 3.86 & 5.83 \\
\hline Late viability & - & 29 & - & 0.10 & 8.28 & 7.58 & 2.95 & 3.36 \\
\hline
\end{tabular}

both viability traits was significantly larger in the inbred lines $(P<0.01)$. As heritability values should lie between 0 and 1 , anomalous estimates were set to the nearest bound. This procedure results in biased estimates of the average realized heritabilities and their sampling errors are reduced (Toro \& Pruñonosa, 1984). Adjusted estimates are shown in Table 2. Both additive genetic variances and heritabilities were significantly larger in the inbred lines $(P<0.01)$. This result was unaffected when the calculations were based on unadjusted estimates.

The distributions of the within-line additive variance in inbred and noninbred lines are shown in Fig. 1 for each trait. For viability traits, little overlap between these distributions was observed.

\section{Discussion}

Our experimental results can be conveniently divided into two parts, according to the character considered. For both viability traits, the following were observed: (1) considerable inbreeding depression, (2) a very small heritability at $F_{0}=0$, (3) a drastic rise of the within-line additive variance with inbreeding, and (4) a parallel increase of the between-line variance larger than predicted by the additive model. These results are consistent with the genetic variance of viability in the base population being essentially generated by deleterious recessive alleles at low frequencies.

Fecundity was also depressed with inbreeding, but the remaining variables behave in a completely different manner, showing: (1) an intermediate heritability at $F_{0}=0$, and (2) small nonsignificant changes of the within-line additive variance and the betweenline variance with inbreeding. For this trait, it appears that both deleterious recessive alleles and loci with substantial additive effects are segregating in the base population.

In order to obtain a better understanding of the situation, consider the following numerical example. 

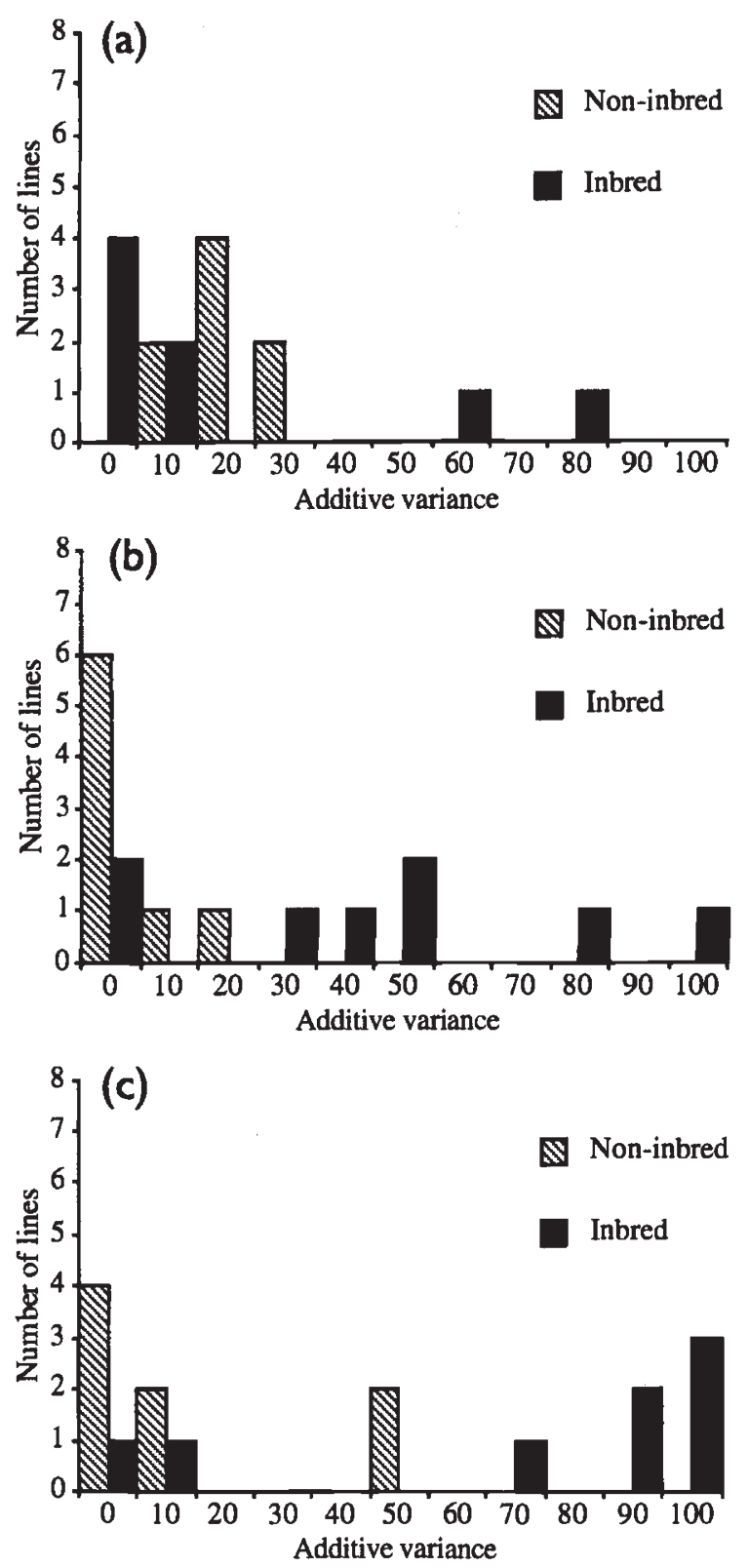

Fig. 1 Distribution of the additive genetic variance in the inbred and noninbred lines of Tribolium castaneum for (a) fecundity, (b) early viability and (c) late viability.

Assume $n_{1}$ additive and $n_{2}$ completely recessive biallelic loci, all of the same effect $(2 a)$ and frequencies $p_{1}$ and $p_{2}$, equal for all loci in each class, respectively. Expected values of the initial additive variance $\left(V_{\mathrm{A} 0}\right)$ and the inbreeding depression $D$ at $F=1 / 4$ are given by (Falconer, 1989)

$V_{\mathrm{A} 0}=n_{1}\left(2 a^{2} p_{1} q_{1}\right)+n_{2}\left(8 a^{2} p_{2} q_{2}^{3}\right)$

and

$D=n_{2} a p_{2} q_{2} / 2$
Values satisfying eqns 1 and 2 for the observed $V_{\mathrm{A} 0}$ and $D$ values and also giving a good fit between expected values at $F=1 / 4$ (Crow \& Kimura, 1970) and those obtained for the within-line additive $\left(V_{\mathrm{AF}}\right)$ and between-line $\left(V_{\mathrm{BF}}\right)$ variances, both expressed as proportions of the additive variance at $F=0$, are given in Table 3 together with the corresponding observations. The number of loci involved $\left(n_{1}\right.$ and $n_{2}$ ) is inversely proportional to the value of $a$. Those in Table 3 have been calculated for $a=\sqrt{V_{\mathrm{P0}}} / 2$.

Thus, 29 equivalent recessive loci with standardized effect equal to one and the recessive allele segregating at low initial frequency, satisfactorily explain the viability results. For fecundity, however, a number of additive loci at intermediate frequencies has to be assumed in addition to deleterious recessives at low frequencies. Alternatively, a comparable fit can be obtained for small frequencies at both classes of loci, implying a large increase of $n_{2}$. Of course, this numerical example only shows the internal coherence of our qualitative interpretation of the data and does not provide quantitative estimates of relevant parameters.

Epistasis could also result in an increase of the within-line additive variance with inbreeding (Goodnight, 1988). Although we unequivocally detected dominance effects from inbreeding depression, our experimental design does not allow a separate study of epistatic effects. Therefore, this discussion has been framed in the simplest terms, i.e. in the absence of nonallelic interactions.

In Tribolium castaneum, previous estimates of relevant parameters for fecundity and viability have only been reported for the Consejo population (Orozco \& Tagarro, 1969; López-Fanjul \& Jódar, 1977) and are in good agreement with those obtained in the present experiment. Thus, both traits showed inbreeding depression. Furthermore, the heritability of viability was small and nonsignificant $(0.17 \pm 0.09)$ and that of fecundity ranged between 0.14 and 0.54 . However, these values are likely to be overestimates as they have been obtained from fullsib correlation analysis. Therefore, in our numerical example the relative contribution of additive loci may be inflated if our estimate of heritability can be considered abnormally high.

In Drosophila melanogaster, inbreeding and artificial selection experiments have also been carried out to investigate the genetic properties of fecundity and viability (Rose, 1984; López-Fanjul \& Villaverde, 1989; García et al., 1994). For both traits, the results are compatible with the genetic variance in populations being generated by segregation at a few loci 
with substantial additive effects and a number of deleterious recessives at low initial frequencies.

Finally, the relevance of the observed redistribution of the genetic variance with inbreeding must be considered. In the inbred lines, higher responses to selection for increasing viability were obtained, but they were accompanied by only a partial recovery (about 50 per cent) of the inbreeding depression incurred. Thus, the significance of this phenomenon on the outcome of natural or artificial selection is uncertain. On the other hand, the observed increment of the between-line variance with inbreeding emphasizes the greater evolutionary potential of small populations postulated by the shifting balance theory. The development of artificial selection strategies making efficient use of the conversion of nonadditive variance into additive variance by means of inbreeding requires further investigation and experimental validation (see Toro, 1993, for a review).

\section{Acknowledgements}

We thank A. Caballero, A. Gallego and A. GarcíaDorado for helpful discussions and comments on earlier drafts of this manuscript. Luis M. Martín kindly allowed us to use his unpublished fecundity data. This work was supported by a grant from the Dirección General de Investigación Científica y Técnica (PB92-1099).

\section{References}

BRYANT, E. H., McCOMMAS, S. A. AND COMBS, L. M. 1986. The effect of an experimental bottleneck upon quantitative genetic variation in the housefly. Genetics, 114, 1191-1211.

CARSON, H. L. 1990. Increased genetic variance after a population bottleneck. Trends Ecol. Evol., 5, 228-230.

CHAPCO, w. 1979. Correlations between chomosome segments and fitness in Drosophila melanogaster. II. The X chromosome and egg viability. Genetics, 92, 595-601.

CROW, J. F. AND KIMURA, M. 1970. An Introduction to Population Genetics Theory. Harper and Row, New York.

FALCONER, D. S. 1989. Introduction to Quantitative Genetics, 3rd edn. Longman, Harlow, Essex.
GARCÍA, N., LÓPEZ-FANJUL, C. AND GARCÍA-DORADO, A. 1994. The genetics of viability in Drosophila melanogaster: effects of inbreeding and artificial selection. Evolution, 48, 1277-1285.

GOODNIGHT, C. J. 1987. On the effect of founder events on epistatic genetic variance. Evolution, 41, 80-91.

GOODNIGHT, C. J. 1988. Epistasis and the effect of founder events on the additive genetic variance. Evolution, 42, 441-454.

LóPEZ-FANıUL, C. 1989. Tests of theory by artificial selection experiments. In: Hill, W. G. and Mackay, T. F. C. (eds) Evolution and Animal Breeding, pp. 129-133. CAB International, Wallingford, U.K.

LÓPEZ-FANJUL, C. AND JÓDAR, B. 1977. The genetic properties of egg laying of virgin females of Tribolium castaneum. Heredity, 39, 251-258.

LÓPEZ-FANJUL, C. AND VILLAVERDE, A. 1989. Inbreeding increases genetic variance for viability in Drosophila melanogaster. Evolution, 43, 1800-1804.

LYNCH, M. 1988. Design and analysis of experiments on random drift and inbreeding depression. Genetics, 120, 791-807.

OROZCO, F. AND TAGARRO, M. P. 1969. Respuesta a la selección y parámetros genéticos de la puesta de huevos en una estirpe de Tribolium castaneum. Boletín INLA, 29, 203-220.

ROBERTSON, A. 1952. The effect of inbreeding on the variation due to recessive genes. Genetics, 37, 189-207.

ROSE, M. R. 1984. Artificial selection on a fitness-component in Drosophila melanogaster. Evolution, 38, 516-526.

TACHIDA, H. AND COCKERHAM, C. C. 1989. Effects of identity disequilibrium and linkage on quantitative variation in finite populations. Genet. Res., 53, 63-70.

TORO, M. A. 1993. A new method aimed at using the dominance variance in closed breeding populations. Génét. Sél. Ev́ol., 25, 63-74.

TORO, M. A. AND PRUÑONOSA, J. V. 1984. The use of prior information in the estimation of heritability by parentoffspring regression. Genetics, Selection, Evolution, 16, 177-184.

WADE, M. J. AND GOODNIGHT, C. J. 1991. Wright's shifting balance theory: an experimental study. Science, 253, 1015-1018.

WRIGHT, s. 1951. The genetical structure of populations. Ann. Eugen., 15, 323-335.

WRIGHT, s. 1978. Evolution and the Genetics of Populations, vol. 3, Experimental Results and Evolutionary Deductions. University of Chicago Press, Chicago, IL. 